\title{
Biochemical Evaluation of a Series of Synthetic Chalcone and Hydrazide Derivatives as Novel Inhibitors of Cruzain from Trypanosoma cruzi
}

\author{
Deise M. Borchhardt,${ }^{a}$ Alessandra Mascarello, ${ }^{b}$ Louise Domeneghini Chiaradia, ${ }^{b}$ \\ Ricardo J. Nunes, ${ }^{b}$ Glaucius Oliva, ${ }^{a}$ Rosendo A. Yunes ${ }^{*, b}$ and Adriano D. Andricopulo ${ }^{*, a}$ \\ ${ }^{a}$ Laboratório de Química Medicinal e Computacional, Centro de Biotecnologia Molecular Estrutural, \\ Instituto de Física de São Carlos, Universidade de São Paulo, 13566-970 São Carlos-SP, Brazil \\ ${ }^{b}$ Laboratório Estrutura e Atividade, Centro de Ciências Físicas e Matemáticas, Departamento de \\ Química, Universidade Federal de Santa Catarina, 88040-900 Florianópolis-SC, Brazil
}

\begin{abstract}
A doença de Chagas, uma infecção parasitária amplamente distribuída na América Latina, é um problema grave de saúde pública com consequiências devastadoras em termos de morbidade e mortalidade humana. A enzima cruzaína é a principal cisteíno protease do Trypanosoma cruzi, agente etiológico da tripanossomíase Americana ou doença de Chagas, e foi selecionada como alvo atrativo para o desenvolvimento de novos fármacos tripanocidas. No presente trabalho, a síntese e os efeitos inibitórios de uma série de trinta e três chalconas e sete hidrazidas são descritos contra a enzima cruzaína de T.cruzi. A maioria dos compostos mostraram inibição promissora in vitro (valores de $\mathrm{IC}_{50}$ na faixa de $20-60 \mu \mathrm{M}$ ), o que sugere o potencial desses compostos como candidatos a líderes para contínuo desenvolvimento. Doze compostos são inéditos, sendo que quatro destes $(7,13,16$ e 18) estão entre os inibidores mais potentes da série.
\end{abstract}

Chagas' disease, a parasitic infection widely distributed throughout Latin America, is a major public health problem with devastating consequences in terms of human morbidity and mortality. The enzyme cruzain is the major cysteine protease from Trypanosoma cruzi, the etiologic agent of American trypanosomiasis or Chagas' disease, and has been selected as an attractive target for the development of novel trypanocidal drugs. In the present work, we describe the synthesis and inhibitory effects of a series of thirty-three chalcone and seven hydrazide derivatives against the enzyme cruzain from T. cruzi. Most of the compounds showed promising in vitro inhibition $\left(\mathrm{IC}_{50}\right.$ values in the range of $20-60 \mu \mathrm{M}$ ), which suggest the potential of these compounds as lead candidates for further development. Twelve compounds have not been reported before, and four of them $(\mathbf{7}, \mathbf{1 3}, 16 \mathrm{e} \mathrm{18})$ are among the most potent inhibitors of the series.

Keywords: chalcones, Trypanosoma cruzi, cruzain, Chagas' disease, inhibition

\section{Introduction}

Chagas' disease, caused by the protozoan parasite Trypanosoma cruzi, is a major cause of illness, morbidity, long-term disability, and death in Latin America. It is estimated that about 16-18 million people are infected and other 100 million are at risk of infection, with more than 50,000 deaths each year. In spite of the alarming health, economic, and social consequences of this parasitic infection, the limited existing drug therapy (nifurtimox and benznidazole) suffers from a combination of drawbacks

*e-mail: aandrico@if.sc.usp.br; ryunes@qmc.ufsc.br including poor efficacy, and serious side effects. Therefore, there is an urgent need for new chemotherapeutic agents with novel mechanisms of action. ${ }^{1-3}$

Enzymes are extremely attractive targets for small molecule drug intervention in human diseases. ${ }^{1,2}$ One important drug target is the major cysteine protease from T. cruzi, cruzain (EC 3.4.22.51). This enzyme is implicated in several vital processes of the parasite, playing a pivotal role during the infection of host cells, replication, and metabolism. The identification and design of potent and selective cruzain inhibitors is, therefore, of great importance. About one decade ago, in a review of inhibitors of cysteine proteases, Otto and Schirmeister ${ }^{4}$ cited a series of chalcone 
derivatives as inhibitors of malarial cysteine proteases. However, the potential of this class of inhibitors has not been fully explored, in spite of its synthetic versatility and potential low toxicity. ${ }^{5}$ To the best of our knowledge, only two chalcones were reported as inhibitors of $T$. cruzi cruzain. ${ }^{6}$ In that respect, there is a justifiable interest in developing structure-activity relationships (SAR) for this class of compounds. On the other hand, acylhydrazides (a class of compounds structurally related to chalcones) have been successfully investigated against $T$. cruzi cruzain. ${ }^{7}$ In addition, both chalcone and acylhydrazide derivatives were active in vitro against $T$. brucei and T. cruzi ${ }^{8,9}$

As part of our research program aimed at discovering novel T. cruzi cruzain inhibitors, we have synthesized and evaluated a series of chalcones and hydrazides. ${ }^{10-12}$

\section{Results and Discussion}

Synthesis

The chalcone derivatives (Table 1) were prepared by aldolic condensation of aromatic aldehydes and corresponding acetophenone (Scheme 1), with yields ranging from 18 to $92 \% .{ }^{12}$ Chalcones $\mathbf{1}$ and $\mathbf{2}$ are derived from 3,4-methylenedioxybenzaldehyde; chalcones 3-6 are derived from 2-hydroxy-3-bromo-4,6- dimethoxyacetophenone (prepared as previously described, with yield of 95\%); ${ }^{13}$ chalcones 7-20 are derived from 3,4-methylenedioxyacetophenone; chalcones 21-27 are derived from 2-naphthylacetophenone; chalcones 28-31 are derived from 2,4,5-trimethoxyacetophenone (prepared as previously described, with yield of $81 \%$ ); ${ }^{14}$ and chalcones $\mathbf{3 2}$ and $\mathbf{3 3}$ are derived from 2,4-dimethoxyacetophenone. The benzylated vanillin (3-methoxy-4-(phenylmethoxy)benzaldehyde) used to prepare chalcone $\mathbf{3 3}$ was obtained according to the procedure of Tsai and Klinman, ${ }^{15}$ with yield of $88 \%$.

The hydrazides (34-40) were prepared by condensation of the obtained 3,4,5-trimethoxybenzohydrazide (41) (previously prepared as described, with yield of $80 \%)^{16}$ and corresponding aromatic aldehydes, with yields ranging from 61 to $72 \%$ (Scheme 2). ${ }^{17}$

All synthesized compounds, including those that had been previously reported (chalcones 1, 2, 4, 5, 8-12, 14, $17,19,21,23-26,28,31-33$; and hydrazides $34,35,38$ and 40) ${ }^{18,19}$ were well characterized by ${ }^{1} \mathrm{H}$ NMR, ${ }^{13} \mathrm{C}$ NMR, IR and elemental analysis. Detailed spectral characterization ( ${ }^{1} \mathrm{H}$ NMR, ${ }^{13} \mathrm{C}$ NMR, IR and elemental analysis) for all novel compounds $(3,6,7,13,15,16,18,20,22,27,29$, 30, 36, 37 and 39) is presented in the experimental part. ${ }^{1} \mathrm{H}$ NMR spectra revealed that all the structures are configured $\mathrm{E}\left(J_{\mathrm{H \alpha}-\mathrm{H} \beta} 15-16 \mathrm{~Hz}\right)$.

\begin{tabular}{|c|c|c|c|c|c|}
\hline Compd. & $\mathrm{R}$ & $\mathrm{R}^{\prime}$ & Compd. & $\mathrm{R}$ & $\mathrm{R}^{\prime}$ \\
\hline 1 & 4-Br-Ph & 3,4-methylenedioxy-Ph & 2 & 2-OH-Ph & 3,4-methylenedioxy-Ph \\
\hline 3 & $2-\mathrm{OH}-3-\mathrm{Br}-4,6-\mathrm{OCH}_{3}-\mathrm{Ph}$ & 1-naphthyl & 4 & $2-\mathrm{OH}-3-\mathrm{Br}-4,6-\mathrm{OCH}_{3}-\mathrm{Ph}$ & $3-\mathrm{NO}_{2}-\mathrm{Ph}$ \\
\hline 5 & $2-\mathrm{OH}-3-\mathrm{Br}-4,6-\mathrm{OCH}_{3}-\mathrm{Ph}$ & $4-\mathrm{OCH}_{3}-\mathrm{Ph}$ & 6 & $2-\mathrm{OH}-3-\mathrm{Br}-4,6-\mathrm{OCH}_{3}-\mathrm{Ph}$ & $4-\mathrm{O}\left(\mathrm{CH}_{2}\right)_{3} \mathrm{CH}_{3}-\mathrm{Ph}$ \\
\hline 7 & 3,4-methylenedioxy-Ph & 2,6-Cl-Ph & 8 & 3,4-methylenedioxy- $\mathrm{Ph}$ & $4-\mathrm{Br}-\mathrm{Ph}$ \\
\hline 9 & 3,4-methylenedioxy-Ph & $4-\mathrm{OCH}_{3}-\mathrm{Ph}$ & 10 & 3,4-methylenedioxy-Ph & $2,4,5-\mathrm{OCH}_{3}-\mathrm{Ph}$ \\
\hline 11 & 3,4-methylenedioxy-Ph & $3,4,5-\mathrm{OCH}_{3}-\mathrm{Ph}$ & 12 & 3,4-methylenedioxy-Ph & $4-\mathrm{CH}_{3}-\mathrm{Ph}$ \\
\hline 13 & 3,4-methylenedioxy-Ph & 1-naphthyl & 14 & 3,4-methylenedioxy-Ph & $\mathrm{Ph}$ \\
\hline 15 & 3,4-methylenedioxy-Ph & 2-Cl-Ph & 16 & 3,4-methylenedioxy-Ph & $2-\mathrm{CH}_{3}$-furan \\
\hline 17 & 3,4-methylenedioxy-Ph & $3-\mathrm{OCH}_{3}-\mathrm{Ph}$ & 18 & 3,4-methylenedioxy-Ph & tiophene \\
\hline 19 & 3,4-methylenedioxy-Ph & $3-\mathrm{OCH}_{3}-4-\mathrm{OBn}-\mathrm{Ph}$ & 20 & 3,4-methylenedioxy-Ph & $4-\mathrm{O}\left(\mathrm{CH}_{2}\right)_{3} \mathrm{CH}_{3}-\mathrm{Ph}$ \\
\hline 21 & 2-naphthyl & $4-\mathrm{OCH}_{3}-\mathrm{Ph}$ & 22 & 2-naphthyl & $2,6-\mathrm{OCH}_{3}-\mathrm{Ph}$ \\
\hline 23 & 2-naphthyl & $2,4,5-\mathrm{OCH}_{3}-\mathrm{Ph}$ & 24 & 2-naphthyl & 4-F-Ph \\
\hline 25 & 2-naphthyl & 3,4-methylenedioxy-Ph & 26 & 2-naphthyl & 2-naphthyl \\
\hline 27 & 2-naphthyl & $2-\mathrm{CH}_{3}$-furan & 28 & $2,4,5-\mathrm{OCH}_{3}-\mathrm{Ph}$ & $2,4,5-\mathrm{OCH}_{3}-\mathrm{Ph}$ \\
\hline 29 & $2,4,5-\mathrm{OCH}_{3}-\mathrm{Ph}$ & 1-naphthyl & 30 & $2,4,5-\mathrm{OCH}_{3}-\mathrm{Ph}$ & tiophene \\
\hline 31 & $2,4,5-\mathrm{OCH}_{3}-\mathrm{Ph}$ & $3-\mathrm{OCH}_{3}-\mathrm{Ph}$ & 32 & $2,4-\mathrm{OCH}_{3}-\mathrm{Ph}$ & $2,4,5-\mathrm{OCH}_{3}-\mathrm{Ph}$ \\
\hline 33 & $2,4-\mathrm{OCH}_{3}-\mathrm{Ph}$ & $3-\mathrm{OCH}_{3}-4-\mathrm{OBn}-\mathrm{Ph}$ & & & \\
\hline
\end{tabular}

Scheme 1. Synthesis of chalcones 1-33. a: KOH $50 \%$ m/v, methanol, rt, $24 \mathrm{~h}$. 


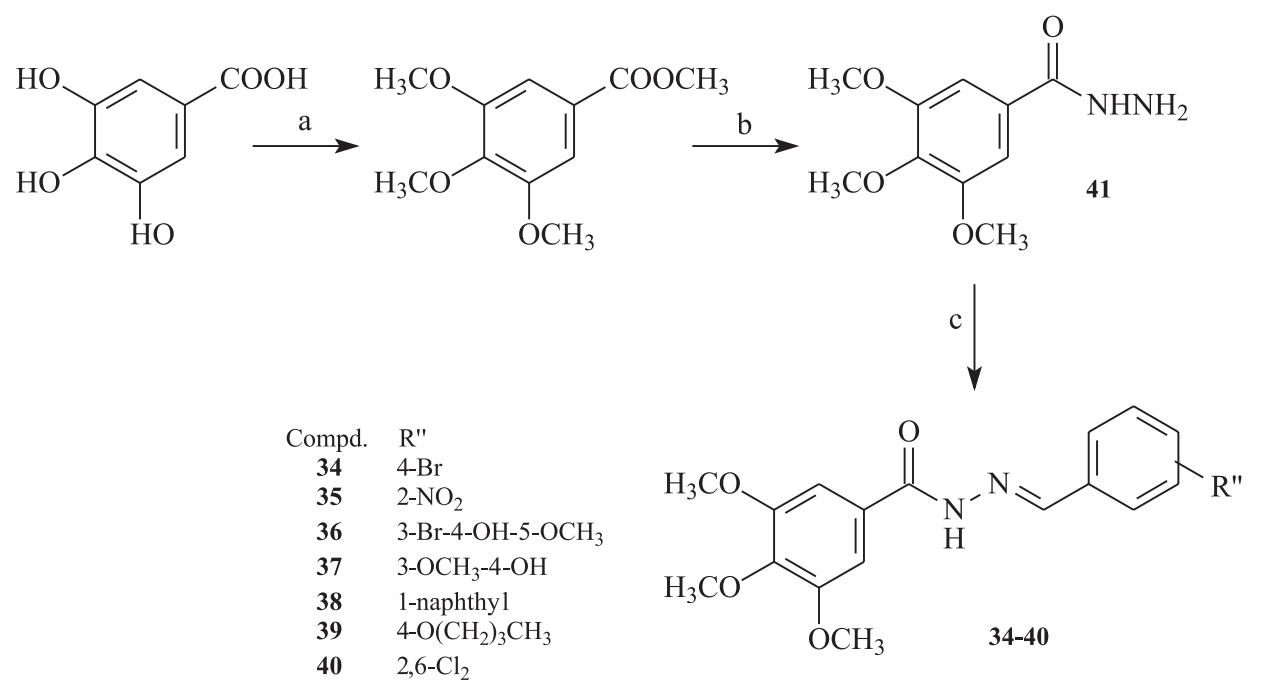

Scheme 2. Synthesis of 3,4,5-trimethoxybenzohydrazide and hydrazide derivatives 34-40. a: $\left(\mathrm{CH}_{3}\right)_{2} \mathrm{SO}_{4}, \mathrm{~K}_{2} \mathrm{CO}_{3}$, acetone, TBAI, reflux, $12 \mathrm{~h}$. b: $\mathrm{H}_{2} \mathrm{NNH} \mathrm{H}_{2}$. $\mathrm{H}_{2} \mathrm{O}, \mathrm{MeOH}$, reflux, 5 h. c: respective aldehyde, $\mathrm{MeOH}$, reflux, $2 \mathrm{~h}$.

\section{Biochemical evaluation of the synthetic compounds}

As can be seen (Table 1), among the 40 derivatives evaluated against $T$. cruzi cruzain, compounds 1, 3-10, 12-14, 16, 18, 20, 21, 25, 28-30, 33-36, and 38-40 have $\mathrm{IC}_{50}$ values in the range of $20-100 \mu \mathrm{M}$.

The $\mathrm{IC}_{50}$ values of compounds 15, 22, 26, $27\left(\mathrm{IC}_{50 \mathrm{~s}}>\right.$ $25 \mu \mathrm{M}), \mathbf{1 1}, \mathbf{1 7}, \mathbf{1 9}, \mathbf{2 3}, \mathbf{2 4}$, and $37\left(\mathrm{IC}_{50 \mathrm{~s}}>50 \mu \mathrm{M}\right)$, as well as of compounds $\mathbf{2 , 3 1}$ and $\mathbf{3 2}$ (the weakest inhibitors of the series with $\mathrm{IC}_{50 \mathrm{~s}}>100 \mu \mathrm{M}$ ), could not be determined accurately due to solubility limitations that appeared to decrease the sensitivity of the assay. The most potent inhibitors of the series, $\mathbf{1}, \mathbf{5}, \mathbf{7}, \mathbf{1 0}, \mathbf{1 3}, \mathbf{1 6}$, and $\mathbf{1 8}\left(\mathrm{IC}_{50}\right.$ values between 20 and $25 \mu \mathrm{M}$ ) represent a new class of lead candidates for further development. Furthermore, with a single exception of compound $\mathbf{5}$, all chalcone derivatives possess the methylenedioxyphenyl (1,3-benzodioxole) moiety, which suggests a significant contribution of this group to the observed activity. In addition, it is worth noting that chalcones 7, 13, 16 and 18 are newly synthesized compounds and are among the most promising compounds for further SAR studies. On the other hand, the hydrazide derivatives (34-40) investigated in this work showed only moderate inhibitory potency, with $\mathrm{IC}_{50}$ values in the range of $40-60 \mu \mathrm{M}$.

\section{Conclusions}

A series of chalcones and hydrazides have been prepared and evaluated in vitro against cruzain from T. cruzi. Most compounds showed promising inhibitory activity against the parasite enzyme, with $\mathrm{IC}_{50}$ values in the low micromolar range. Our results demonstrate the potential of these compounds as a new class of small molecule inhibitors of $T$. cruzi cruzain. Future efforts to optimize these compounds will involve the design, synthesis and SAR studies of a variety of more watersoluble derivatives, as well as investigation about the importance of methylenedioxyphenyl moiety side in the structure of chalcones through the determination of the possible mechanism of enzyme inhibition and inhibitor binding mode. Efforts in this direction are in progress, and will be reported in due course.

\section{Experimental}

\section{Physicochemical data of the synthesized compounds}

The purified compounds were obtained in yields ranging from 18 to $92 \%$. Melting points were determined with a Microquímica MGAPF-301 apparatus and are uncorrected. IR spectra were recorded with an Abb Bomen FTLA 2000 spectrometer on $\mathrm{KBr}$ disks. NMR $\left({ }^{1} \mathrm{H}\right.$ and ${ }^{13} \mathrm{C}$ NMR) were recorded on a Varian Oxford AS-400 (400 MHz) spectrometer, using tetramethylsilane as an internal standard. Elemental analysis was carried out with a CHNS EA 1110. Percentages of $\mathrm{C}$ and $\mathrm{H}$ were in agreement with the product formula (within $+0.4 \%$ of theoretical values of C).

General procedure for the synthesis and purification of chalcones (1-33)

The chalcones were synthesized as shown in Scheme 1. All reagents used in this work were analytical grade and were purchased from Merck and Sigma-Aldrich. Exceptions 
Table 1. Structures and $\mathrm{IC}_{50}$ values of a series of synthetic chalcone and hydrazide derivatives as inhibitors of $T$. cruzi cruzain

\begin{tabular}{|c|c|c|c|c|c|c|c|c|}
\hline Compd. & Structure & $\mathrm{IC}_{50}{ }^{\mathrm{a}}(\mu \mathrm{M})$ & Compd. & Structure & $\mathrm{IC}_{50}{ }^{\mathrm{a}}(\mu \mathrm{M})$ & Compd. & Structure & $\mathrm{IC}_{50}{ }^{\mathrm{a}}(\mu \mathrm{M})$ \\
\hline 1 & & 20 & 2 & & $>100$ & 3 & & 37 \\
\hline 4 & & 45 & 5 & & 20 & 6 & & 82 \\
\hline 7 & & 20 & 8 & & 30 & 9 & & 35 \\
\hline 10 & & 25 & 11 & & $>50$ & 12 & & 30 \\
\hline 13 & & 25 & 14 & & 50 & 15 & & $>25$ \\
\hline 16 & & 23 & 17 & & $>50$ & 18 & & 22 \\
\hline 19 & & $>50$ & 20 & & 46 & 21 & & 60 \\
\hline 22 & & $>25$ & 23 & & $>50$ & 24 & & $>50$ \\
\hline 25 & & 50 & 26 & & $>25$ & 27 & & $>25$ \\
\hline 28 & & 100 & 29 & & 48 & 30 & & 100 \\
\hline 31 & & $>100$ & 32 & & $>100$ & 33 & & 37 \\
\hline 34 & & 55 & 35 & & 60 & 36 & & 50 \\
\hline 37 & & $>50$ & 38 & & 40 & 39 & & 47 \\
\hline 40 & & 50 & & & & & & \\
\hline
\end{tabular}

${ }^{\mathrm{a}} \mathrm{IC}_{50}$ values are representative of three independent experiments performed in triplicate and the standard deviations were within $10 \%$ of the mean values reported for each compound. 
are 2-hydroxy-3-bromo-4,6-dimethoxyacetophenone, 2,4,5-trimethoxyacetophenone and 3-methoxy-4(phenylmethoxy)-benzaldehyde, which were prepared as previously described. ${ }^{13-15}$ The chalcones (1-33) were prepared by magnetic stirrer of the acetophenone derivative $(1.2 \mathrm{mmol})$, methanol $(20 \mathrm{~mL}), \mathrm{KOH} 50 \% \mathrm{~m} / \mathrm{v}(5 \mathrm{~mL})$, and the corresponding aldehyde $(1.2 \mathrm{mmol})$, at room temperature for $24 \mathrm{~h}$. Distilled water and hydrochloric acid $(10 \%)$ were added to the reaction for total precipitation of the products. The compounds were then obtained by vacuum filtration and later recrystallized from dichloromethane and hexane. Chalcones 1, 2, 4, 5, 8-12, 14, 17, 19, 21, 23-26, 28 , 31-33 were previously described in the literature, ${ }^{18}$ while compounds $3,6,7,13,15,16,18,20,22,27,29$ and 30 are novel compounds which have not been reported. The chalcones (3-6) showed yields in the range of $40-50 \%$ due the presence of the bromine atom in the starting material acetophenone, which stabilizes the enolate intermediate of the reaction making it less reactive. This effect was not observed for the chalcones (7-20), which showed yields in the range of $70-98 \%$.

(2E)-1-(2'-Hydroxy, 3'-bromo, 4',6'-dimethoxyphenyl)-3(1-naphthyl)-2-propen-1-one (3)

Yellow solid; mp $218-219^{\circ} \mathrm{C}$; yield: $42 \%$; ${ }^{1} \mathrm{H}$ NMR (acetone- $\left.\mathrm{d}_{6}\right) \delta 4.06\left(\mathrm{~s}, 3 \mathrm{H}, o-\mathrm{OCH}_{3}\right), 4.14\left(\mathrm{~s}, 3 \mathrm{H}, p-\mathrm{OCH}_{3}\right)$, $6.51(\mathrm{~s}, 1 \mathrm{H}, \mathrm{H} 5$ ') 7.60 (d, 1H, J7.6 Hz, H10), $7.61(\mathrm{~m}, \mathrm{H} 5)$, 7.62 (d, J 7.6 Hz, H8), 7.70 (t, 1H, J 7.6 Hz, H9), 8.03 (m, $3 \mathrm{H}, \mathrm{H} 4, \mathrm{H} 6), 8.09$ (d, 1H, J $15.4 \mathrm{~Hz}, \mathrm{H \alpha}$ ), 8.38 (d, 1H, J 8.4 $\mathrm{Hz}, \mathrm{H} 3), 8.66(\mathrm{~d}, 1 \mathrm{H}, J 15.4 \mathrm{~Hz}, \mathrm{H} \beta) ;{ }^{13} \mathrm{C} \mathrm{NMR}(\mathrm{CDCl} 3)$ $\delta 56.39$ ( $p$-OCH3), 56.62 (o-OCH3), 87.41 (C3’), 92.24 (C5'), 107.20 (C1'), 123.90 (Co), 125.46 (C10), 125.68 (C3), 126.52 (C9), 127.15 (C5), 128.96 (C4), 129.79 (C8), 130.86 (C6), 132.00 (C2), 133.00 (C7), 133.97 (C1), 140.42 (Cß), 162.27 (C6'), 162.55 (C2'), 163.46 (C4'), 192.91 $(\mathrm{C}=\mathrm{O}) ; \mathrm{IR}(\mathrm{KBr}) v_{\max } / \mathrm{cm}^{-1}: 3438(\mathrm{OH}), 1624,1218(\mathrm{C}=\mathrm{O})$, $1552(\mathrm{C}=\mathrm{C}), 1347,1040$ (C-O), 2952, 1415, 1314, 1250, 1129, 973, 800, 767, 659 (Ar); Anal. calc. for $\mathrm{C}_{21} \mathrm{H}_{17} \mathrm{BrO} 4$ : C 61.03; H 4.15. Found: C 59.99; H 4.01.

(2E)-1-(2'-Hydroxy-3'-bromo-4',6'-dimethoxyphenyl)-3(4-buthoxyphenyl)-2-propen-1-one (6)

Yellow solid; mp 172-173 ${ }^{\circ} \mathrm{C}$; yield: $47 \%$; ${ }^{1} \mathrm{H}$ NMR $\left(\mathrm{CDCl}_{3}\right) \delta 0.98\left(\mathrm{t}, 3 \mathrm{H},-\mathrm{CH}_{3}\right), 1.47-1.60\left(\mathrm{~m}, 2 \mathrm{H},-\mathrm{CH}_{2} \mathrm{CH}_{3}\right)$, 1.75-1.81 (m, $\left.2 \mathrm{H},-\mathrm{CH}_{2} \mathrm{CH}_{2} \mathrm{CH}_{3}\right), 3.98\left(\mathrm{~s}, 3 \mathrm{H}, \mathrm{OCH}_{3}\right), 3.99$ (s, $\left.3 \mathrm{H}, \mathrm{OCH}_{3}\right), 4.01\left(\mathrm{t}, 2 \mathrm{H},-\mathrm{OCH}_{2}-\right), 6.06(\mathrm{~s}, 1 \mathrm{H}, \mathrm{H} 5$ '), $6.91(\mathrm{~d}, 2 \mathrm{H}, J 8.4 \mathrm{~Hz}, \mathrm{H} 3, \mathrm{H} 5), 7.54$ (d, 2H, J $8.4 \mathrm{~Hz}, \mathrm{H} 2$, H6), $7.75(\mathrm{~d}, 1 \mathrm{H}, J 15.6 \mathrm{~Hz}, \mathrm{H \alpha}), 7.83(\mathrm{~d}, 1 \mathrm{H}, J 15.6 \mathrm{~Hz}$, $\mathrm{H} \beta), 14.96(\mathrm{OH}) ;{ }^{13} \mathrm{C}$ NMR (acetone- $\left.d_{6}\right) \delta 13.42\left(\mathrm{CH}_{3}\right)$, $19.18\left(-\mathrm{CH}_{2} \mathrm{CH}_{3}\right), 31.28\left(-\mathrm{CH}_{2} \mathrm{CH}_{2} \mathrm{CH}_{3}\right), 56.25\left(\mathrm{OCH}_{3}\right)$, $67.86\left(-\mathrm{OCH}_{2}{ }^{-}\right), 88.37\left(\mathrm{C}^{\prime}\right), 94.61\left(\mathrm{C}^{\prime}\right), 104.43\left(\mathrm{Cl}^{\prime}\right)$,
115.19 (C3, C5), 124.46 (C $\alpha), 128.04$ (C1), 130.71 (C2, C6), 143.72 (C $\beta), 160.02$ (C4), 168.46 (C2'), 168.82 (C6'), 176.12 (C4'), $198.07(\mathrm{C}=\mathrm{O})$; IR (KBr) $v_{\max } / \mathrm{cm}^{-1} 3450$, 2945, 1615, 1554, 1221, 963; Anal. Calc. for $\mathrm{C}_{21} \mathrm{H}_{23} \mathrm{BrO}_{5}$ : C 57.94; H 5.33. Found: C 57.27; H 5.27.

(2E)-1-(1,3-Benzodioxol-5-yl)-3-(2,6-dichlorophenyl)-2propen-1-one (7)

Light yellow solid; mp $118-119{ }^{\circ} \mathrm{C}$; yield: $90 \%$; ${ }^{1} \mathrm{H}$ NMR $\left(\mathrm{CDCl}_{3}\right) \delta 6.08$ (s, 2H, - $\left.\mathrm{OCH}_{2} \mathrm{O}-\right), 6.90(\mathrm{~d}, 1 \mathrm{H}$, $J 8.0 \mathrm{~Hz}, \mathrm{H} 5$ ') 7.21 (t, 1H, J 7.6 Hz, H4), 7.38 (d, 2H, J $8.4 \mathrm{~Hz}, \mathrm{H} 3, \mathrm{H} 5), 7.54$ (s, 1H, H2'), 7.62 (d, 1H, J 16.0

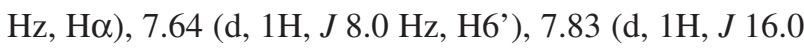
$\mathrm{Hz}, \mathrm{H} \beta) ;{ }^{13} \mathrm{C} \mathrm{NMR}\left(\mathrm{CDCl}_{3}\right) \delta 102.16\left(-\mathrm{OCH}_{2} \mathrm{O}-\right), 108.23$ (C5'), 108.76 (C2'), 125.40 (C6'), 128.00 (C $\alpha$ ), 129.07 (C3, C5), 129.99 (C1'), 130.49 (C4), 132.75 (C6), 132.98 (C2), 135.40 (C1), 137.50 (C 3$), 148.62$ (C3'), 152.23 (C4'), $188.22(\mathrm{C}=\mathrm{O})$; IR $(\mathrm{KBr}) v_{\max } / \mathrm{cm}^{-1} 1655,1242(\mathrm{C}=\mathrm{O}), 1595$ $(\mathrm{C}=\mathrm{C}), 1310,1033$ (C-O), 2905, 1503, 1439, 1107, 979, 927, 786, 743 (Ar); Anal. calc. for $\mathrm{C}_{16} \mathrm{H}_{10} \mathrm{Cl}_{2} \mathrm{O}_{3}$ : C 59.84; H 3.14. Found: C 59.69; H 3.26.

(2E)-1-(1,3-Benzodioxol-5-yl)-3-(1-naphthyl)-2-propen1-one (13)

Yellow solid; mp 137-139 ${ }^{\circ} \mathrm{C}$; yield: $91 \%$; ${ }^{1} \mathrm{H}$ NMR $\left(\mathrm{CDCl}_{3}\right) \delta 6.09$ (s, 2H, - $\left.\mathrm{OCH}_{2} \mathrm{O}-\right), 6.93(\mathrm{~d}, 1 \mathrm{H}, J 8.0 \mathrm{~Hz}$, H5'), $7.53(\mathrm{~d}, 1 \mathrm{H}, J 16.0 \mathrm{~Hz}, \mathrm{H \alpha}), 7.54(\mathrm{~d}, 1 \mathrm{H}, J 8.0 \mathrm{~Hz}$, H10), 7.58 (m, 1H, H4), 7.59 (d, 1H, J 8.0 Hz, H6'), 7.61 (s, 1H, H2'), 7.61 (m, 1H, H5), 7.72 (dd, 1H, J 8.0/1.0 Hz, H9), 7.90 (m, 1H, H8), 7.93 (d, 1H, J 8.0 Hz, H6), 8.28 (d, $1 \mathrm{H}, J 8.0 \mathrm{~Hz}, \mathrm{H} 3), 8.66(\mathrm{~d}, 1 \mathrm{H}, J 16.0 \mathrm{~Hz}, \mathrm{H} \beta) ;{ }^{13} \mathrm{C} \mathrm{NMR}$ $\left(\mathrm{CDCl}_{3}\right) \delta 102.13\left(-\mathrm{OCH}_{2} \mathrm{O}-\right), 108.19\left(\mathrm{C}^{\prime}\right), 108.73\left(\mathrm{C}^{\prime}{ }^{\prime}\right)$, 109.99 (C10), 123.80 (C6'), 124.63 (C $\alpha), 125.05$ (C3), 125.23 (C9), 125.66 (C5), 126.52 (C4), 127.17 (C8), 128.96 (C6), 130.90 (C2), 131.99 (C1'), 133.77 (C7), $133.96(\mathrm{C} 1)$, $141.46(\mathrm{C} \beta), 148.60$ (C3'), $153.81\left(\mathrm{C}^{\prime}\right), 188.29(\mathrm{C}=\mathrm{O})$; IR (KBr) $v_{\text {max }} / \mathrm{cm}^{-1} 1646,1253(\mathrm{C}=\mathrm{O}), 1584(\mathrm{C}=\mathrm{C}), 1343$, 1037 (C-O), 2919, 1498, 1444, 1307, 1115, 978, 930, 806, 773, 732, 709 (Ar); Anal. calc. for $\mathrm{C}_{20} \mathrm{H}_{14} \mathrm{O}_{3}: \mathrm{C}$ 79.46; $\mathrm{H}$ 4.67. Found: C 78.53; H 4.80.

(2E)-1-(1,3-Benzodioxol-5-yl)-3-(2-chlorophenyl)-2propen-1-one (15)

Cream solid; mp $112-113{ }^{\circ} \mathrm{C}$; yield: $87 \%$; ${ }^{1} \mathrm{H}$ NMR $\left(\mathrm{CDCl}_{3}\right) \delta 6.08\left(\mathrm{~s}, 2 \mathrm{H},-\mathrm{OCH}_{2} \mathrm{O}-\right), 6.90(\mathrm{~d}, 1 \mathrm{H}, J 8.0 \mathrm{~Hz}$, H5'), 7.31 (m, 1H, H4), 7.34 (m, 1H, H5), $7.44(\mathrm{~m}, 1 \mathrm{H}$,

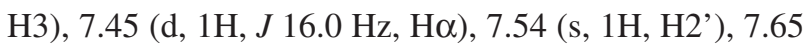
(dd, 1H, J 8.0/1.0 Hz, H6') 7.74 (m, 1H, H6), 8.16 (d, 1H, $J 16.0 \mathrm{~Hz}, \mathrm{H} \beta) ;{ }^{13} \mathrm{C} \mathrm{NMR}\left(\mathrm{CDCl}_{3}\right) \delta 101.91\left(-\mathrm{OCH}_{2} \mathrm{O}-\right)$, 107.94 (C2'), 124.52 (C6'), 124.89 (C $\alpha), 127.04$ (C5), 127.75 (C6), 130.30 (C3), 131.03 (C4), 132.72 (C1'), 
133.38 (C1), 135.43 (C2), 140.06 (Cß), 148.36 (C3'), 151.85 (C4'), $188.15(\mathrm{C}=\mathrm{O})$; IR ( $\mathrm{KBr}) v_{\max } / \mathrm{cm}^{-1} 1653$, $1241(\mathrm{C}=\mathrm{O}), 1598(\mathrm{C}=\mathrm{C}), 1319,1037(\mathrm{C}-\mathrm{O}), 2900,1489$, 1435, 1106, 975, 924, 813, 751, 569 (Ar); Anal. calc. for $\mathrm{C}_{16} \mathrm{H}_{11} \mathrm{ClO}_{3}$ : C 67.03; H 3.87. Found: C 67.14; H 4.05.

(2E)-1-(1,3-Benzodioxol-5-yl)-3-(5-methylfuran-2-yl)-2propen-1-one (16)

Light orange solid; mp $98-99^{\circ} \mathrm{C}$; yield: $87 \%$; ${ }^{1} \mathrm{H}$ NMR $\left(\mathrm{CDCl}_{3}\right) \delta 2.39(\mathrm{CH} 3), 6.06\left(\mathrm{~s}, 2 \mathrm{H},-\mathrm{OCH}_{2} \mathrm{O}-\right), 6.13(\mathrm{~d}$, $1 \mathrm{H}, J 4.0 \mathrm{~Hz}, \mathrm{H} 4), 6.61$ (d, 1H, J 4.0 Hz, H5), 6.89 (d, 1H, $J 8.0 \mathrm{~Hz}, \mathrm{H} 5$ '), 7.33 (d, 1H, J $16.0 \mathrm{~Hz}, \mathrm{H \alpha}), 7.52(\mathrm{~d}, 1 \mathrm{H}$, $J 16.0 \mathrm{~Hz}, \mathrm{H} \beta$ ), 7.67 (d, 1H, J 8.0 Hz, H6'), 7.54 (s, 1H, $\mathrm{H} 2$ '); ${ }^{13} \mathrm{C} \mathrm{NMR}\left(\mathrm{CDCl}_{3}\right) \delta 14.25\left(\mathrm{CH}_{3}\right), 102.02\left(-\mathrm{OCH}_{2} \mathrm{O}-\right)$, 108.10 (C2'), 108.60 (C5'), 109.52 (C4), 117.46 (C5), 118.21 (C6'), $124.73(\mathrm{C} \alpha), 130.71(\mathrm{C} \beta, \mathrm{C} 1), 133.34\left(\mathrm{C} 1{ }^{\prime}\right)$, 148.44 (C3'), 150.64 (C1), 151.73 (C4'), 155.95 (C3), $187.98(\mathrm{C}=\mathrm{O})$; IR ( $\mathrm{KBr}) v_{\max } / \mathrm{cm}^{-1} 1651,1251(\mathrm{C}=\mathrm{O})$, 1601 (C=C), 1360, 1036 (C-O), 2908, 1567, 1492, 1438, 1291, 1192, 1107, 1010, 994, 936, 906, 846, 792, 612, 539 (Ar); Anal. calc. For $\mathrm{C}_{15} \mathrm{H}_{12} \mathrm{O}_{4}$ : C 70.31; H 4.72. Found: C 70.09; H 4.79 .

(2E)-1-(1,3-Benzodioxol-5-yl)-3-(thiophen-2-yl)-2-propen1-one (18)

Beige solid; mp 101-103 ${ }^{\circ} \mathrm{C}$; yield: $91 \%$; ${ }^{1} \mathrm{H}$ NMR $\left(\mathrm{CDCl}_{3}\right) \delta 6.07$ (s, 2H, - $\left.-\mathrm{OCH}_{2} \mathrm{O}-\right), 6.90(\mathrm{~d}, 1 \mathrm{H}, J 4.0 \mathrm{~Hz}$, H5'), 7.09 (d, 1H, J 8.0 Hz, H4), 7.29 (d, 1H, J $16.0 \mathrm{~Hz}$, $\mathrm{H \alpha}$ ), 7.35 (d, 1H, J 4.0 Hz, H5), 7.41 (d, 1H, J $4.0 \mathrm{~Hz}, \mathrm{H} 3)$, $7.52 \mathrm{~s}(\mathrm{H} 2$ '), 7.63 (d, 1H, J 8.0 Hz, H6'), 7.93 (d, 1H, J 16.0 $\mathrm{Hz}, \mathrm{H} \beta) ;{ }^{13} \mathrm{C} \mathrm{NMR}\left(\mathrm{CDCl}_{3}\right) \delta 102.09\left(-\mathrm{OCH}_{2} \mathrm{O}-\right), 108.16$ (C2'), 108.59 (C5'), 120.68 (\$C6'), 124.81 (C 2 ), 128.54 (C4), 128.79 (C5), 132.13 (C 3$), 133.12$ (C1'), 136.94 (C3), 140.72 (C1), 148.52 (C3'), 151.93 (C4'), 187.87 (C=O); IR $(\mathrm{KBr}) v_{\max } / \mathrm{cm}^{-1} 1644,1264(\mathrm{C}=\mathrm{O}), 1581(\mathrm{C}=\mathrm{C}), 1361$, 1040 (C-O), 1500, 1443, 1325, 1301, 1202, 1109, 960, 910, 799, 697, 567 (Ar); Anal. calc. for $\mathrm{C}_{14} \mathrm{H}_{10} \mathrm{O}_{3} \mathrm{~S}: \mathrm{C} 65.10 ; \mathrm{H}$ 3.90. Found: C 64.73; H 3.63.

(2E)-1-(1,3-Benzodioxol-5-yl)-3-(4-buthoxyphenyl)-2propen-1-one (20)

Cream solid; mp 102-104 ${ }^{\circ} \mathrm{C}$; yield: $92 \%$; ${ }^{1} \mathrm{H}$ NMR $\left(\mathrm{CDCl}_{3}\right) \delta 0.99\left(\mathrm{t}, 3 \mathrm{H}, J 8.0 \mathrm{~Hz}, \mathrm{CH}_{3}\right), 1.51\left(\mathrm{~m},-\mathrm{CH}_{2}-\mathrm{CH}_{3}\right)$, $1.79\left(\mathrm{~m},-\mathrm{CH}_{2}-\mathrm{CH}_{2}-\mathrm{CH}_{3}\right), 4.01\left(\mathrm{t}, 2 \mathrm{H}, J 8.0 \mathrm{~Hz},-\mathrm{O}-\mathrm{CH}_{2}-\right)$, 6.06 (s, 2H, -OCH2O-), 6.89 (d, 1H, J $8.0 \mathrm{~Hz}, \mathrm{H} 5$ '), 6.92 (d, $1 \mathrm{H}, J 8.0 \mathrm{~Hz}, \mathrm{H} 3, \mathrm{H} 5), 7.37$ (d, 1H, J $16.0 \mathrm{~Hz}, \mathrm{H \alpha}$ ), 7.53 (s, 1H, H2'), 7.58 (d, 1H, J 8.0 Hz, H2, H6), 7.65 (d, $1 \mathrm{H}, J 8.0 \mathrm{~Hz}, \mathrm{H6}$ '), 7.77 (d, 1H, J 16.0 Hz, Hß); ${ }^{13} \mathrm{C} \mathrm{NMR}$ $\left(\mathrm{CDCl}_{3}\right) \delta 13.84\left(\mathrm{CH}_{3}\right), 19.22\left(-\mathrm{CH}_{2}-\mathrm{CH}_{3}\right), 31.21\left(-\mathbf{C H}_{2}-\right.$ $\left.\mathrm{CH}_{2}-\mathrm{CH}_{3}\right), 67.87\left(-\mathrm{O}-\mathrm{CH}_{2}-\right), 101.81$ (-OCH $\left.2 \mathrm{O}-\right), 107.87$ (C2'), 108.44 (C5'), 114.88 (C3, C5), 119.18 (C6'), 124.46
(C $\alpha), 127.46$ (C1), $130.14\left(\mathrm{C} 1^{\prime}, \mathrm{C} 2, \mathrm{C} 6\right), 144.25(\mathrm{C} \beta)$, 148.22 (C3'), 152.11 (C4'), 161.22 (C4), $181.79(\mathrm{C}=\mathrm{O})$; IR (KBr) $v_{\text {max }} / \mathrm{cm}^{-1} 1654,1240(\mathrm{C}=\mathrm{O}), 1596(\mathrm{C}=\mathrm{C}), 1330$, 1030 (C-O), 2953, 2863, 1504, 1438, 1291, 1173, 1110 , 973, 924, 807, 571 (Ar); Anal. calc. for $\mathrm{C}_{20} \mathrm{H}_{20} \mathrm{O}_{4}$ : C 74.06; H 6.21. Found: C 74.28; H 6.46.

(2E)-1-(2-Naphthyl)-3-(2,6-dimethoxyphenyl)-2-propen1-one (22)

Cream solid; mp 109- $11{ }^{\circ} \mathrm{C}$; yield: $79 \%$; ${ }^{1} \mathrm{H}$ NMR $\left(\mathrm{CDCl}_{3}\right) \delta 3.94\left(\mathrm{~s}, 6 \mathrm{H}, \mathrm{OCH}_{3}\right), 6.61(\mathrm{~d}, 1 \mathrm{H}, J 8.0 \mathrm{~Hz}, \mathrm{H} 3$, H5), 7.31 (t, $1 \mathrm{H}, J 8.0 \mathrm{~Hz}, \mathrm{H} 4), 7.55$ (dd, $1 \mathrm{H}, J 8.0 / 1.0 \mathrm{~Hz}$, H5'), 7.60 (dd, 1H, J 8.0/1.0 Hz, H6'), 7.90 (d, 1H, J 8.0 Hz, H4'), 7.93 (d, 1H, J 8.0 Hz, H10'), 8.00 (d, 1H, J 8.0 Hz, H9'), 8.11 (d, 1H, J 8.0 Hz, H7'), 8.13 (d, 1H, J 16.0 $\mathrm{Hz}, \mathrm{H \alpha}$ ), 8.33 (d, $1 \mathrm{H}, J 16.0 \mathrm{~Hz}, \mathrm{H} \beta), 8.53$ (s, 1H, H2'); ${ }^{13} \mathrm{C}$ NMR $\left(\mathrm{CDCl}_{3}\right) \delta 56.15$ (OCH3), 104.00 (C3, C5), 113.19 (C1), 125.08 (C $\alpha$ ), 125.40 (C10'), 126.76 (C5'), 128.01 (C7'), 128.25 (C9'), 128.51 (C6'), 129.76 (C2'), 130.06 (C4), 131.76 (C4'), 132.84 (C8'), 135.52 (C1'), 136.07 (Cß), 136.53 (C3'), 160.62 (C2, C6), $192.40(\mathrm{C}=\mathrm{O}) . \mathrm{IR}$ $(\mathrm{KBr}) v_{\text {max }} / \mathrm{cm}^{-1} 1645,1256(\mathrm{C}=\mathrm{O}), 1581(\mathrm{C}=\mathrm{C}), 1320,1011$ (C-O), 2934, 2833, 1470, 1439, 1177, 1110, 851, 757, 618, 535, 459 (Ar); Anal. calc. for $\mathrm{C}_{21} \mathrm{H}_{18} \mathrm{O}_{3}: \mathrm{C}$ 79.23; H 5.70. Found: C 79.14; H 5.69.

(2E)-1-(2-Naphthyl)-3-(5-methylfuran-2-yl)-2-propen-1one (27)

Gold yellow solid; mp $85-87^{\circ} \mathrm{C}$; yield: $92 \%$; ${ }^{1} \mathrm{H}$ NMR $\left(\mathrm{CDCl}_{3}\right) \delta 2.43\left(\mathrm{~s}, 3 \mathrm{H}, \mathrm{CH}_{3}\right), 6.15(\mathrm{~d}, 1 \mathrm{H}, J 4.0 \mathrm{~Hz}, \mathrm{H} 4)$, 6.66 (d, 1H, J 4.0 Hz, H5), 7.54 (d, 1H, J $16.0 \mathrm{~Hz}, \mathrm{H \alpha}$ ), 7.58 (m, 1H, H5'), 7.61 (m, 1H, H6'), 7.62 (d, 1H, J 16.0 $\mathrm{Hz}, \mathrm{H} \beta), 7.90$ (d, 1H, J 8.0 Hz, H4'), 7.94 (d, 1H, J $8.0 \mathrm{~Hz}$, H10'), 8.01 (d, 1H, J 8.0 Hz, H9'), 8.12 (d, 1H, J $8.0 \mathrm{~Hz}$, $\mathrm{H} 7$ '), 8.57 (s, $1 \mathrm{H}, \mathrm{H} 2$ '); ${ }^{13} \mathrm{C} \mathrm{NMR}\left(\mathrm{CDCl}_{3}\right) \delta 14.09\left(\mathrm{CH}_{3}\right)$, 109.43 (C4), 117.52 (C5), 118.36 (C10'), 124.51 (C $\alpha$ ), 126.67 (C5'), 127.80 (C7'), 128.25 (C9'), 128.46 (C6'), $129.56\left(\mathrm{C}^{\prime}\right), 129.76\left(\mathrm{C} 4^{\prime}\right), 130.80(\mathrm{C} \beta), 132.63$ (C8'), 135.43 (C1'), 135.75 (C3'), 150.45 (C1), 155.96 (C3), $189.69(\mathrm{C}=\mathrm{O}) ; \mathrm{IR}(\mathrm{KBr}) v_{\max } / \mathrm{cm}^{-1} 1648,1284(\mathrm{C}=\mathrm{O}), 1557$ $(\mathrm{C}=\mathrm{C}), 1362,1012(\mathrm{C}-\mathrm{O}), 3056,1595,1182,1114,819$, 780, 710, 614, 535, 473 (Ar); Anal. calc. for $\mathrm{C}_{18} \mathrm{H}_{14} \mathrm{O}_{2}$ : C 82.42; H 5.38. Found: C 81.68; H 5.30.

(2E)-1-(2',4',5'-Trimethoxyphenyl)-3-(1-naphthyl)-2propen-1-one (29)

Yellow solid; mp 162-164 ${ }^{\circ} \mathrm{C}$; yield: $87 \%$; ${ }^{1} \mathrm{H}$ NMR $\left(\mathrm{CDCl}_{3}\right) \delta 3.90\left(\mathrm{~s}, 3 \mathrm{H}, o-\mathrm{OCH}_{3}\right), 3.92\left(\mathrm{~s}, 3 \mathrm{H}, m-\mathrm{OCH}_{3}\right)$, 3.98 (s, 3H, $p-\mathrm{OCH}_{3}$ ), 6.56 (s, 1H, H3'), 7.45 (s, 1H, H6'), 7.51 (t, 1H, J $8.0 \mathrm{~Hz}, \mathrm{H} 9), 7.53$ (m, 1H, H5), 7.59 (d, 1H, H4), $7.72(\mathrm{~d}, 1 \mathrm{H}, J 16.0 \mathrm{~Hz}, \mathrm{H \alpha}), 7.85$ (d, 1H, J 8.0 Hz, 
H8), 7.88 (m, 2H, H6, H10), 8.31 (d, 1H, J $8.0 \mathrm{~Hz}, \mathrm{H} 3$ ), $8.55(\mathrm{~d}, 1 \mathrm{H}, J 16.0 \mathrm{~Hz}, \mathrm{H} \beta) ;{ }^{13} \mathrm{C} \mathrm{NMR}\left(\mathrm{CDCl}_{3}\right) \delta 56.37$ $\left(o-\mathrm{OCH}_{3}\right), 56.49\left(m-\mathrm{OCH}_{3}\right), 57.01\left(p-\mathrm{OCH}_{3}\right), 97.26\left(\mathrm{C}^{\prime}\right)$, 113.50 (C6'), 120.67 (C1'), 123.99 (Co), 125.21 (C10), 125.67 (C3), 125.95 (C9), 126.23 (C5), 128.87 (C4), 130.11 (C8), 130.36 (C6), 132.03 (C2), 133.96 (C7), 138.96 (C $\beta)$, 141.46 (C5'), 143.67 (C1), 153.94 (C2'), 155.18 (C4'), $191.12(\mathrm{C}=\mathrm{O}) ; \mathrm{IR}(\mathrm{KBr}) v_{\max } / \mathrm{cm}^{-1} 3456,774(\mathrm{C}-\mathrm{S}), 1660$, $1214(\mathrm{C}=\mathrm{O}), 1610(\mathrm{C}=\mathrm{C}), 1321,1027(\mathrm{C}-\mathrm{O}), 2834,1516$, $846(\mathrm{Ar})$.

(2E)-1-(2',4',5'-Trimethoxyphenyl)-3-(thiophen-2-yl)-2propen-1-one (30)

Yellow solid; mp 117-119 ${ }^{\circ} \mathrm{C}$; yield: $86 \%$; ${ }^{1} \mathrm{H}$ NMR $\left(\mathrm{CDCl}_{3}\right) \delta 3.90\left(\mathrm{~s}, 3 \mathrm{H}, o-\mathrm{OCH}_{3}\right), 3.94\left(\mathrm{~s}, 3 \mathrm{H}, m-\mathrm{OCH}_{3}\right)$, 3.96 (s, 3H, p- $\left.-\mathrm{OCH}_{3}\right), 6.54$ (s, 1H, H3'), 7.07 (t, 1H, J 4.0 $\mathrm{Hz}, \mathrm{H} 4), 7.31$ (d, 1H, J 4.0 Hz, H5), 7.37 (d, 1H, J $4.0 \mathrm{~Hz}$,

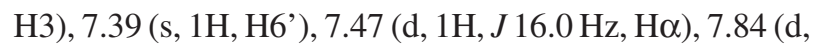
$1 \mathrm{H}, J 16.0 \mathrm{~Hz}, \mathrm{H} \beta) ;{ }^{13} \mathrm{C} \mathrm{NMR}\left(\mathrm{CDCl}_{3}\right) \delta 56.37\left(o-\mathrm{OCH}_{3}\right)$, $56.45\left(m-\mathrm{OCH}_{3}\right), 57.01\left(p-\mathrm{OCH}_{3}\right), 97.33\left(\mathrm{C}^{\prime}\right), 113.38$ (C6'), 120.56 (C1'), 126.51 (Co), 128.09 (C5), 128.41 (C4), 131.31 (C3), 134.63 (C1', C 3$), 141.38$ (C5'), 153.88 (C2'), 155.13 (C4'), $189.21(\mathrm{C}=\mathrm{O})$; IR $(\mathrm{KBr}) \mathrm{v}_{\max } / \mathrm{cm}^{-1}$ : 3099, 729 (C-S), 1651, $1267(\mathrm{C}=\mathrm{O}), 1606(\mathrm{C}=\mathrm{C}), 1307$, 1023 (C-O), 2997, 2940, 2829, 1582, 1504, 1406, 1206, $1137,847,510(\mathrm{Ar})$.

General procedure for the synthesis and purification of 3,4,5-trimethoxybenzohydrazide $(\mathbf{4 1})^{16}$

3,4,5-trimethoxybenzohydrazide was obtained from a mixture of gallic acid $(6 \mathrm{mmol})$, dimethyl sulphate (28 mmol), $\mathrm{K}_{2} \mathrm{CO}_{3}(26 \mathrm{mmol})$ and tetrabutylammonium iodide (TBAI) $(0.1 \mathrm{~g})$ in acetone $(30 \mathrm{~mL})$. The mixture was refluxed for $12 \mathrm{~h}$, after which the precipitate was filtered and washed with acetone. The ester obtained $(6 \mathrm{mmol})$ was treated with a solution of $99 \% \mathrm{~N}_{2} \mathrm{H}_{4}(4.4 \mathrm{mmol})$ in methanol $(20 \mathrm{~mL})$ and refluxed for $5 \mathrm{~h}$ and then kept at room temperature overnight. The solid obtained was filtered and recrystallized from hot methanol to afford the 3,4,5-trimethoxybenzohydrazide (80\%).

General procedure for the synthesis and purification of $N^{\prime}-$ substituted benzylidene-3,4,5-trimethoxybenzohydrazide $(34-40)^{17}$

The hydrazides were synthesized as shown in Scheme 2. 3,4,5-trimethoxybenzohydrazide ( $2 \mathrm{mmol}$ ) was mixed with the appropriate aldehyde $(2 \mathrm{mmol})$ in methanol $(15 \mathrm{~mL})$ and refluxed for $2 \mathrm{~h}$. After cooling, the crude product was collected by filtration, washed and recrystallized from hot ethanol to give white solids. Hydrazides $\mathbf{3 4}, \mathbf{3 5}, \mathbf{3 8}$ and $\mathbf{4 0}$ were previously described in the literature, ${ }^{19}$ while $\mathbf{3 6}, \mathbf{3 7}$ and $\mathbf{3 9}$ are novel compounds which have not been reported.

(2E)-N'-(3'-Bromo-4'-hydroxy-5'-methoxybenzilidene)3,4,5-trimethoxybenzohydrazide (36)

White solid, mp 244-250 ${ }^{\circ} \mathrm{C}$; yield: $72 \%$; ${ }^{1} \mathrm{H}$ NMR $\left(\mathrm{DMSO}-d_{6}\right) \delta 3.73\left(\mathrm{~s}, 3 \mathrm{H}, m-\mathrm{OCH}_{3}\right), 3.87\left(\mathrm{~s}, 6 \mathrm{H}, m-\mathrm{OCH}_{3}\right)$, 3.90 (s, 3H, p- $\left.\mathrm{OCH}_{3}\right), 7.22$ (s, 2H, H2, H6), 7.33 (s, 1H, H2'), 7.43 (s, 1H, H6'), 8.33 (s, 1H, -N=CH), 10.03 (br, $1 \mathrm{H}, \mathrm{OH}), 11.68$ (s, 1H, -CONH); ${ }^{13} \mathrm{C}$ NMR (DMSO- $\left.d_{6}\right)$ $\delta 56.78\left(m-\mathrm{OCH}_{3}\right), 56.72\left(p-\mathrm{OCH}_{3}\right), 60.81\left(m-\mathrm{OCH}_{3}\right)$, 105.85 (C2, C6), 108.88 (C6'), 110.03 (C3'), 125.05 (C1), 127.30 (C2'), 129.29 (C1'), 142.10 (C4), 146.49 (-N=CH), 147.46 (C4'), 149.32 (C5'), 153.37 (C3, C5), 163.16 (-CONH); IR (KBr) $v_{\max } / \mathrm{cm}^{-1} 3364(\mathrm{~N}-\mathrm{H}), 1646$, $1251(\mathrm{C}=\mathrm{O}), 1569(\mathrm{C}=\mathrm{N}), 1309,1027$ (C-O), 2985, 1505, 1426, 1296, 1143, 1002 (Ar).

(2E)-N'-(4'-Hydroxy-5'-methoxybenzilidene)-3,4,5trimethoxybenzohydrazide (37)

White solid; mp $198{ }^{\circ} \mathrm{C}$; yield: $72 \%$; ${ }^{1} \mathrm{H}$ NMR $\left(\mathrm{DMSO}_{-} d_{6}\right) \delta 3.71\left(\mathrm{~s}, 3 \mathrm{H}, m-\mathrm{OCH}_{3}\right), 3.82(\mathrm{~s}, 6 \mathrm{H}$, $\left.m-\mathrm{OCH}_{3}\right), 3.85\left(\mathrm{~s}, 3 \mathrm{H}, p-\mathrm{OCH}_{3}\right), 6.84(\mathrm{~d}, 1 \mathrm{H}, J 8.0$ Hz, H3'), 7.08 (d, 1H, J $8.0 \mathrm{~Hz}, \mathrm{H} 2$ ') , 7.20(s, 2H, H2, H6), 7.30 (s, 1H, H6'), 8.34 (s, 1H, -N=CH), $9.55(\mathrm{br}, 1 \mathrm{H}, \mathrm{OH}), 11.52(\mathrm{~s}, 1 \mathrm{H},-\mathrm{CONH}) ;{ }^{13} \mathrm{C} \mathrm{NMR}$ $\left(\mathrm{DMSO}_{6}\right) \delta 56.25\left(p-\mathrm{OCH}_{3}\right), 56.77\left(m-\mathrm{OCH}_{3}\right), 60.81$ $\left(m-\mathrm{OCH}_{3}\right), 105.78\left(\mathrm{C}^{2}, \mathrm{C} 6\right), 109.64\left(\mathrm{C}^{\prime}\right), 116.15$ (C3'), 122.83 (C2'), 126.36 (C1'), 129.45 (C1), 140.96 (C4), 148.74 (-N=CH), 149.08 (C4'), 149.73 (C5'), 153.37 (C3, C5), 162.99 (-CONH); IR (KBr) $v_{\max } / \mathrm{cm}^{-1}$ $3418(\mathrm{~N}-\mathrm{H}), 1664,1230(\mathrm{C}=\mathrm{O}), 1584(\mathrm{C}=\mathrm{N}), 1338,1030$ (C-O), 3265, 2885, 1612, 1495, 1411, 1097 (Ar).

(2E)-N'-(4'-buthoxybenzilidene)-3,4,5-trimethoxybenzohydrazide (39)

White solid; mp $119^{\circ} \mathrm{C}$; yield: $61 \%$; ${ }^{1} \mathrm{H}$ NMR $\left(\mathrm{CDCl}_{3}\right)$ $\delta 0.96(\mathrm{t}, \mathrm{CH} 3), 1.47\left(\mathrm{~m},-\mathrm{CH}_{2}-\mathrm{CH}_{3}\right), 1.71\left(\mathrm{~m},-\mathrm{CH}_{2}-\mathrm{CH}_{2}-\mathrm{CH}_{3}\right)$, $4.04\left(\mathrm{t},-\mathrm{O}-\mathrm{CH}_{2}-\right), 3.39\left(\mathrm{~s}, 9 \mathrm{H}, m-\mathrm{OCH}_{3}\right), 3.45(\mathrm{~s}, 3 \mathrm{H}$, $\left.p-\mathrm{OCH}_{3}\right), 3.85\left(\mathrm{~s}, 3 \mathrm{H}, \mathrm{OCH}_{3}\right), 7.04(\mathrm{~d}, 2 \mathrm{H}, J 8.0 \mathrm{~Hz}$, H3', H5'), 7.24 (s, 2H, H2, H6), 7.68 (d, 2H, J $8.0 \mathrm{~Hz}$, H2', H6'), 8.41 (s, 1H, -N=CH), 11.61 (s, 1H, -CONH); ${ }^{13} \mathrm{C} \mathrm{NMR}\left(\mathrm{CDCl}_{3}\right) \delta 14.38\left(\mathrm{CH}_{3}\right), 19.41\left(-\mathrm{CH}_{2}-\mathrm{CH}_{3}\right), 31.36$ $\left(-\mathrm{CH}_{2}-\mathrm{CH}_{2}-\mathrm{CH}_{3}\right), 56.78\left(m-\mathrm{OCH}_{3}\right), 60.83\left(p-\mathrm{OCH}_{3}\right)$, 68.02 (-O-CH2-), 105.80 (C2, C6), 115.50 (C3', C5'), 127.37 (C1'), 129.35 (C1), 129.40 (C2', C6'), 148.41 $(-\mathrm{N}=\mathrm{CH}), 153.37$ (C3, C5), $161.03\left(\mathrm{C}^{\prime}\right), 163.07(\mathrm{C}=\mathrm{O})$; IR (KBr) $v_{\max } / \mathrm{cm}^{-1} 3236(\mathrm{~N}-\mathrm{H}), 1652,1237(\mathrm{C}=\mathrm{O}), 1577$ $(\mathrm{C}=\mathrm{N}), 1330,1027$ (C-O), 2961, 2875, 1509, 1413, 1237, 1123, 998, 848 (Ar). 


\section{Biochemical evaluation of the synthetic compounds}

\section{Materials}

Cruzain truncated in the C-terminal extension was obtained from Escherichia coli (strain M15 or DH5 $\alpha$ containing the expression plasmid) following the previously reported procedure. ${ }^{20}$ All reagents for buffer preparation, the standard inhibitor E-64 and the substrate Z-Phe-ArgAMC were purchased from Sigma-Aldrich. Substrate and inhibitors candidates at $10 \mathrm{mM}$ stock solutions in neat DMSO were stored at $-20^{\circ} \mathrm{C}$ and at $-4^{\circ} \mathrm{C}$, respectively.

\section{Procedure}

The activity of the enzyme was measured and quantified through active-site titration with the irreversible inhibitor E-64, as described previously. ${ }^{21}$ For the biochemical evaluation against $T$. cruzi cruzain, the highly purified enzyme $(0.64 \mathrm{nM})$ in $50 \mathrm{mM}$ sodium phosphate, $100 \mathrm{mM}$ sodium chloride, $5 \mathrm{mM}$ EDTA, pH 6.5, containing $5 \mathrm{mM}$ DTT, was incubated with the compounds (chalcones 1-33 and hydrazides 34-40, Table 1) for $5 \mathrm{~min}$ at room temperature followed by the addition of the fluorogenic substrate Z-Phe-Arg-AMC. ${ }^{22}$ Fluorescence was monitored on a Wallac 1420-042 PerkinElmer spectrofluorometer and measurements were done using $355 \mathrm{~nm}$ as the excitation wavelength and $460 \mathrm{~nm}$ as the emission wavelength, as previously described..$^{10}$ Cruzain activity was measured as an increase of fluorescence intensity of liberated aminocoumarin when Z-Phe-Arg-AMC was used as the substrate. The percentage of inhibition was calculated according to the following equation:

$\%$ of Inhibition $=100 \times\left(1-V_{\mathrm{i}} / V_{0}\right)$

where $V_{\mathrm{i}}$ and $V_{0}$ are the initial velocities (enzyme activities) determined in the presence and in the absence of inhibitor, respectively. Values of $\mathrm{IC}_{50}$ were independently determined by making rate measurements for at least six inhibitor concentrations (inhibition range: $20-85 \%$ ). $\mathrm{The} \mathrm{IC}_{50}$ values correspond to the concentration of compound required for $50 \%$ inhibition of cruzain, and were determined from the collected data by nonlinear regression analysis employing the Sigma-Plot enzyme kinetics module.

\section{Acknowledgments}

We gratefully acknowledge financial support from CNPq, FAPESP and CAPES, Brazil. We thank Prof. Ana Paula C. A. Lima from the Federal University of Rio de Janeiro for the T. cruzi cruzain clone and the Department of Chemistry, UFSC, for the chemical analysis.

\section{References}

1. Fujii, N.; Mallari, J. P.; Hansell, E. J.; Mackey, Z.; Doyle, P.; Zhou, Y. M.; Gut, J.; Rosenthal, P. J.; McKerrow, J. H.; Guy, R. K.; Bioorg. Med. Chem. Lett. 2005, 15, 121.

2. Siles, R.; Chen, S. E.; Zhou, M.; Pinney, K. G.; Trawick, M. L.; Bioorg. Med. Chem. Lett. 2006, 16, 4405; Andricopulo, A. D.; Akoachere, M. B.; Krogh, R.; Nickel, C.; McLeish, M. J.; Kenyon, G. L.; Arscott, L. D.; Williams, C. H.; Davioud-Charvet, E.; Becker, K.; Bioorg. Med. Chem. Lett. 2006, 16, 2283.

3. Guido, R. V. C.; Oliva, G.; Montanari, C. A.; Andricopulo, A. D.; J. Chem. Inf. Model. 2008, 48, 918; Cardoso, C. L.; Lima, V. V.; Zottis, A.; Oliva, G.; Andricopulo, A. D.; Wainer, I. W.; Moaddel, R.; Cass, Q. B.; J. Chromatogr. A 2006, 1120, 151.

4. Otto, H. H.; Schirmeister, T.; Chem. Rev. 1997, 97, 133.

5. Lunardi, F.; Guzela, M.; Rodrigues, A. T.; Corrêa, R.; Mangrich, I. E.; Steindel, M.; Grisard, E. C.; Assreuy, J.; Calixto, J. B.; Santos, A. R. S.; Antimicrob. Agents Chemother. 2003, 47, 1449.

6. Li, R.; Chen, X.; Gong, B.; Selzer, P. M.; Li, Z.; Davidson, E.; Kurzban, G.; Miller, R. E.; Nuzum, E. O.; McKerrow, J. H.; Fletterick, R. J.; Gillmor, S. A.; Craik, C. S.; Kuntz, I. D.; Cohen, F. E.; Kenyon, G. L.; Bioorg. Med. Chem. 1996, 4, 1421.

7. Rodrigues, C. R.; Flaherty, T. M.; Springer, C.; McKerrow, J. H.; Cohen, F. E.; Bioorg. Med. Chem. Lett. 2002, 12, 1537.

8. Troeberg, L.; Chen, X.; Flaherty, T. M.; Morty, R. E.; Cheng, M.; Hua, H.; Springer, C.; McKerrow, J. H.; Kenyon, G. L.; Lonsdale-Eccles, J. D.; Coetzer, T. H. T.; Cohen, F. E.; Molec. Med. 2000, 6, 660 .

9. Ramiréz, I.; Carabot, A.; Meléndez, P.; Carmona, J.; Jimenez, M.; Patel, A. V.; Crabb, T. A.; Blunden, G.; Cary, P. D.; Croft, S. L.; Costa, M.; Phytochemistry 2003, 64, 645.

10. Zanatta, N.; Amaral, S. S.; dos Santos, J. M.; de Mello, D. L.; Fernandes, L. S.; Bonacorso, H. G.; Martins, M. A. P.; Andricopulo, A. D.; Borchhardt, D. M.; Bioorg. Med. Chem. 2008, 16, 10236.

11. Guido, R. V. C.; Trossini, G. H. G.; Castilho, M. S.; Oliva, G.; Ferreira, E. I.; Andricopulo, A. D.; J. Enz. Inhib. Med. Chem. 2008, 23, 964.

12. Chiaradia, L. D.; dos Santos, R.; Vitor, C. E.; Vieira, A. A.; Leal, P. C.; Nunes, R. J.; Calixto, J. B.; Yunes, R. A.; Bioorg. Med. Chem. 2008, 16, 658.

13. Cechinel-Filho, V.; Vaz, Z. R.; Zunino, L.; Calixto, J. B.; Yunes, R. A.; Eur. J. Med. Chem. 1996, 31, 833.

14. Högberg, T.; Bengtsson, S.; Paulis, T. de.; Johansson, L.; Ström, P; Hall, H.; Ögren, S. O.; J. Med. Chem. 1990, 33, 1155.

15. Tsai, S.; Klinmam, J. P.; Bioorg. Chem. 2003, 31, 172.

16. Chida, A. S.; Vani, P. V. S. N.; Chandrasekharam, M.; Srinivasan, R.; Singh, A. K.; Synth. Commun. 2001, 31, 657.

17. Jin, L.; Chen, J.; Song, B.; Chen, Z.; Yang, S.; Li, Q.; Hu, D.; Xu, R.; Bioorg. Med. Chem. Lett. 2006, 16, 5036.

18. Basaif, S. A.; Albar, H. A.; Faidallah, H. M.; Indian J. 
Heterocycl. Chem. 1995, 5, 121; Dittmer, C.; Raabe, G.; Hintermann, L.; Eur. J. Org. Chem. 2007, 35, 5886; CamposBuzzi, F.; Campos, J. P.; Tonini, P. P.; Corrêa, R.; Yunes, R. A.; Boeck, P.; Cechinel-Filho, V.; Arch. Pharm. 2006, 339, 361; Ali, S. M.; Iqbal, J.; Ilyas, M.; J. Chem. Res. 1984, 7, 236; Lygo, B.; Gardiner, S. D.; McLeod, M. C.; To, D. C. M.; Org. Biomol. Chem. 2007, 5, 2283; Katritzky, A. R.; Kuanar, M.; Dobchev, D. A.; Vanhoecke, B. W. A.; Karelson, M.; Parmar, V. S.; Stevens, C. V.; Bracke, M. E.; Bioorg.Med. Chem. 2006, 14, 6933; Ishii, H.; Ishikawa, T.; Deushi, T.; Harada, K.; Watanabe, T.; Ueda, E.; Ishida, T.; Sakamoto, M.; Kawanabe, E.; Chem. Pharm. Bull. 1983, 31, 3024; Tully, W.; Main, L.; Nicholson, B. K.; J. Organomet. Chem. 1995, 503, 75; Insuasty, B.; Orozco, F.; Quiroga, J.; Abonia, R.; Nogueras, M.; Cobo, J.; Eur. J. Med. Chem. 2008, 43, 1955; Kunick, C.; Bleeker, C.; Pruehs, C.; Totzke, F.; Schaechtele, C.; Kubbutat, M. H. G.; Link, A.; Bioorg. Med. Chem. Lett. 2006, 16, 2148; Xia, Y.; Yang, Z.-Y.; Xia, P.; Bastow, K. F.; Nakanishi, Y.; Lee, K. H.; Bioorg. Med. Chem. Lett. 2000, 10, 699; Parmar, V. S.; Sharma, S. L.; Bisht, K. S.; Singh, A.; Gupta, S. A.; Jain, R.; Vardhan, A.; Zhurnal Organicheskoi Khimii 1995, 31, 1839; Levai, A.; Jeko, J.; J. Heterocycl. Chem. 2006, 43, 111; Sanchez-Viesca, F.; Ciencia 1973, 27, 75; Rajasekaran, K.; Gnanasekaran, C.; Ind. J. Chem. Sect. A: Inorg. Phys. Theor. Anal. 1986, 25A, 64; Robinson, T. P.; Hubbard, R. B.; Ehlers, T. J.; Arbiser, J. L.; Goldsmith, D.
J.; Bowen, J. P.; Bioorg. Med. Chem. 2005, 13, 4007; Bowen, P. J.; Robinson, T. P.; Ehlers, T.; Goldsmith, D.; Arbiser, J.; PCT Int. Appl. 2001, 90, pp.; Anjaneyulu, A. S. R.; Rani, G. S.; Mallavadhani, U. V.; Murthy, Y. L. N.; Ind. J. Heterocycl. Chem. 1994, 4, 9; Bianco, A.; Cavarischia, C.; Guiso, M.; Eur. J. Org. Chem. 2004, 13, 2894; De Almeida, P. A.; Fraiz, S. V. Jr.; Braz-Filho, R.; J. Braz. Chem. Soc. 1999, 10, 347.

19. Shehata, I. A.; Nasr, M. N.; El-Subbagh, H. I.; Gineinah, M. M.; Kheira, S. M.; Sci. Pharm. 1996, 64, 133; Mazzone, G.; Arrigo, R. R.; Bollettino delle Sedute della Accademia Gioenia di Scienze Naturali in Catania 1971, 10, 689.

20. Eakin, A. E.; Mills, A. A.; Harth, G.; McKerrow, J. H.; Craik, C. S. J.; Biol. Chem. 1992, 267, 7411.

21. Barret, A. J.; Kembhavi, A. A.; Brown, M. A.; Kirschke, H.; Knight, C. G.; Tamai, M.; Hanada, K.; Biochem. J. 1982, 201, 189.

22. Li, R.; Kenyon, G. L.; Cohen, F. E.; Chem, X.; Gong, B.; Dominguez, J. N.; Davidson, E.; Kurzban, G.; Miller, R. E.; Nuzum, E. O.; Rosenthal, P. J.; McKerrow, J. H.; J. Med. Chem. 1995, 38, 5031 .

Received: April 16, 2009

Web Release Date: October 29, 2009

FAPESP helped in meeting the publication costs of this article. 